\title{
Responsabilidad Social Interorganizacional. Un caso de estudio: Abuelos en red
}

\author{
LiLiana GaLÁN* \\ Mauricio Javier Bertonati Horcas** \\ Graciela NAFrIA ${ }^{* * *}$ \\ Argentina
}

\section{Resumen}

El siguiente trabajo presenta la acción conjunta del Banco Supervielle y la Secretaría de Extensión de la Facultad de Ciencias Económicas de la UNLP, ejemplo de participación social, cultural e integración a través de programas de alfabetización informática para mejorar la calidad de vida de los adultos mayores.

\section{Introducción}

En los próximos años, la población de adultos mayores se verá cada vez más limitada para satisfacer sus necesidades de participación en la sociedad de la información, si no se instrumentan acciones educativas

\footnotetext{
* $\quad$ mba Facultad Ciencias Económicas Universidad Nacional de La Plata. Contacto: lgalan@ econo.unlp.edu.ar

* Licenciado en Tecnología Educativa, Universidad Tecnológica Nacional, Facultad Regional Mendoza. Contacto: Mauricio.Bertonati@supervielle.com.ar

**:Licenciada en Administración, Universidad Nacional de La Plata. Contacto: graciela.nafria@econo.unlp.edu.ar
} 
que los incorporen a procesos de enseñanza y aprendizaje impregnados por tecnología que contribuyan a que la sociedad cambie su mirada sobre este grupo etario. Se considera que los programas de alfabetización en el uso de tecnologías digitales pueden aportar a mejorar la calidad de vida de los adultos mayores, abriendo canales efectivos de participación social, cultural y de verdadera integración. En este marco se constituye en objetivo central del presente programa desarrollar un proceso de alfabetización en el uso de tecnologías digitales destinado a adultos mayores. Al respecto, la pregunta se concentra en identificar cuáles son las acciones de responsabilidad social interorganizacional en red para mejorar la calidad de vida de adultos mayores. En esta dirección este trabajo tiene como objetivo, exponer un caso de responsabilidad social interorganizacional, sobre acciones de responsabilidad social en red. La acción conjunta entre el Banco Supervielle y las universidades nacionales de Argentina, en este caso particular, la Facultad de Ciencias Económicas de la Universidad Nacional de La Plata, a través de la Secretaría de Extensión y el Departamento de Tecnologías Informáticas constituye un ejemplo de ello. En tal sentido, el Banco Supervielle implementó a partir del año 2007, a través del área de Responsabilidad Social Corporativa, el Programa denominado "Abuelos en Red", en él se desarrollan talleres gratuitos de capacitación sobre uso de herramientas informáticas básicas orientadas a la comunicación, destinado a personas mayores de 55 años, que sean jubilados o pensionados. Estos talleres se dictan en distintos espacios, algunas sedes funcionan en Centros de Servicios del Banco y otras en universidades asociadas.

\section{Metodología}

La estrategia metodológica se articula en dos ejes interrelacionados: uno se refiere a la Formación de Formadores, y otro a la producción de condiciones que efectivicen el derecho a la alfabetización digital. Este eje metodológico parte de considerar el lugar de los actores en el proyecto, en tanto sujetos socio-históricos que ponen en juego sus competencias de saber hacer para realizar una práctica de vinculación tecnológica. 
Desde el año 2007, el Banco ha capacitado alrededor de 35.000 adultos mayores, en 1200 talleres. En tanto en la Facultad de Ciencias Económicas de la Universidad Nacional de La Plata asistieron, durante los años 2014-2015, aproximadamente 200 adultos mayores en 20 cursos.

Mucho se ha escrito y debatido en los últimos años sobre la responsabilidad social, como una práctica de distintos tipos de organizaciones, públicas como privadas. A pesar de que cuando se aborda la temática, en particular en el caso de las empresas, este concepto deviene justamente de la corresponsabilidad que le cabe al sector privado, público y ONG para satisfacer las demandas de la comunidad, si bien no se registran muchas evidencias de responsabilidad social interorganizacional.

Ya en 2003 Bernardo Kliksberg escribía: "Lo que pueden hacer políticas públicas activas combinadas con el voluntariado, la responsabilidad social empresarial, la acción vecinal, el respaldo de las comunidades religiosas, la contribución de las universidades, es mucho más que los esfuerzos aislados de los actores” (p. 33). Sin embargo, las prácticas formalizadas en Argentina no abundan, tal vez y en términos de hipótesis, por prejuicios existentes en la Universidad Pública respecto a la participación del sector privado en sus acciones y particularmente en la extensión universitaria donde se inserta el programa al que este trabajo hace referencia.

En la actualidad, la concientización creciente de las empresas en cuanto a su responsabilidad social les ha permitido permear las fronteras de su quehacer específico y encontrar al alcance de la mano la oportunidad, de comprometerse con sus públicos, como es el caso del Banco Supervielle, que recibe permanentemente adultos mayores que cobran en sus distintas sucursales su jubilación y les ofrece un camino para mantener sus relaciones perdidas por la ausencia de trabajo a través de convenios con las universidades de Argentina para desarrollar el programa "Abuelos en Red".

Este entramado de relaciones construye lo que aquí se denomina Responsabilidad Social Interorganizacional. Hay referencias en la literatura de la importancia de las redes interorganizacionales para generar capital social. Las redes interorganizacionales presuponen un campo de acción constituido por organizaciones independientes, que se han 
distribuido las tareas y se articulan de una manera tal que obtienen los objetivos compartidos de una política (Jaramillo, 2006). En tal sentido, este trabajo intenta enmarcar lo que se denomina Responsabilidad Social Interorganizacional (RSI) una red vincular entre organizaciones públicas, privadas y/o organizaciones de la sociedad civil, construida para potenciar las capacidades de los participantes en pos de lograr objetivos compartidos de una política de responsabilidad social.

\section{Marco teórico}

En el siglo Xxi han prosperado las redes entre organizaciones debido a la posibilidad que ofrecen las mismas de construir consensos para la acción y complementar los objetivos de cada una de las participantes. Nuevos desafíos en el contexto de la gobernabilidad democrática, la sociedad del conocimiento y la tecnología interpelan a la Universidad en sus distintas funciones, planteando la necesidad de adoptar nuevas estrategias que medien las relaciones entre lo público y lo privado, entre el gobierno y la sociedad.

La articulación de redes de actores sociales e institucionales, como modalidad de concertar estrategias, programas y acciones, se constituye actualmente en un desafío imprescindible para las políticas en el área de extensión. Si esta tarea se lleva cabo en un marco de análisis y discusión amplio y participativo, con intervención de los múltiples actores involucrados, con espíritu tendiente a la búsqueda de consensos, quizás interpretar más cabalmente el presente y diseñar los escenarios del futuro sea más posible y se logren acuerdos de mayor significación. Se trata de la construcción de espacios y mecanismos de articulación entre las instituciones políticas y los diversos actores sociales, mediante la creación de instrumentos y procedimientos puestos a disposición de los ciudadanos y grupos de la sociedad para facilitar su intervención en los asuntos públicos. Particularmente, promover la conformación de una organización en red para llevar adelante acciones, en la cual los actores públicos y privados, las instituciones estatales y no estatales, participan y cooperan en la formulación e implementación de las políticas públicas (Mayntz, 2001). Esto permitirá avanzar en la 
conformación de una nueva institucionalidad orientada a convertir a la gestión pública en un espacio más permeable a las demandas que emergen de la sociedad, y a retirar de este modo al Estado el monopolio exclusivo de la definición de la agenda social (Cunill,1999).

La importancia de la organización en red (Messner, 1995) es que, a partir de ella, se fortalece el tejido social, se promueve la participación popular en la construcción de un proyecto colectivo y una estrategia compartida que contribuya al desarrollo de la comunidad local. En estos fundamentos puede entenderse la conformación de redes interorganizacionales de Responsabilidad Social Interorganizacional (RSI).

Las redes interorganizacionales presuponen un campo de acción constituido por organizaciones independientes, que se han distribuido las tareas y se articulan de una manera tal que obtienen los objetivos compartidos de una política. Esta interacción lleva a encontrar oportunidades que generan valor público de una manera poco costosa. Según Volpentesta (2009), el abanico de actividades de gestión social que pueden emprender las empresas a través de sus acciones de RSE es amplio y diverso. Por tal motivo, cuando se intenta abordar una determinada problemática es difícil que un solo actor opere.

De ahí que aparece como legítima la vinculación de las empresas con otras organizaciones para cumplir con sus objetivos de responsabilidad social. Cuando las otras organizaciones encuentran en el vínculo la oportunidad de satisfacer también sus acciones de responsabilidad social, podría instalarse la idea de Responsabilidad Social Interorganizacional (RSI). En tal sentido, podría encuadrarse el caso que se describe en este marco. En tanto una empresa, el Banco Supervielle se vincula con las universidades a fin de promover la inclusión social de los adultos mayores. Por el lado de las Universidades, particularmente la FCE de la UNLP, encuentra en la ejecución del Programa un eje para el cumplimiento de su Responsabilidad Social Universitaria (OEA-BID, 2007). En efecto, según Valleys (2009) y Valleys (2008), uno de los cuatro ejes de la Responsabilidad Social Universitaria es la Participación Social que consiste en la gestión socialmente responsable de la participación de la universidad en la comunidad. Se trata de "la realización de proyectos con otros actores de tal modo que se constituyan vínculos (capital social) para el aprendizaje mutuo y el 
desarrollo social". La universidad participa en la comunidad, va más allá de capacitar a públicos vulnerables y también constituye comunidades de aprendizaje mutuo para el desarrollo.

\section{La problemática de los adultos mayores}

La vertiginosidad de los cambios acecidos en los últimos años exige contar con herramientas para comprender y actuar en el presente, desde un lugar de integración social, cuestión que constituye una apuesta para la universidad pública en su relación con la sociedad.

Los adultos mayores constituyen un grupo poblacional que no accede a muchos de los bienes y servicios de los que la sociedad dispone. Es decir, no cuenta con las mismas oportunidades que poseen otros sectores de apropiarse de conocimientos que les posibiliten relacionarse y realizar gestiones vinculadas a la resolución de situaciones en la vida cotidiana mediante el uso de tecnología digital.

El programa de referencia interpela y da respuesta a afirmaciones como estas: "En muchos casos, la tecnología el desarrollo no ayudó a solucionar los problemas de las personas, y al contrario, los complicó”, según comentan Camarotta y Beremblum (2009) .Y los autores siguen: "Las mismas instituciones bancarias, en su propósito por simplificar sus procedimientos y agilizar lo que el jubilado necesita, también han caído en errores serios de diseño", afirmación con la cual se refieren al uso de tarjetas electrónicas sin tener en cuenta dos aspectos fundamentales: el rechazo fuerte a la nueva tecnología por parte de esta franja etárea y el interés que reviste para ellos el reunirse con sus pares una vez al mes para intercambiar y vincularse socialmente.

Identificar esta problemática nos lleva a proponer desde el enfoque de derechos, un trabajo de extensión universitaria dirigido a generar condiciones que faciliten la apropiación de conocimientos y herramientas informáticas, desde una práctica de inclusión social, en un mundo globalizado que difiere sustancialmente de la sociedad en la que se educaron y trabajaron. En este sentido, entendemos que la falta accesibilidad a la alfabetización digital de los adultos mayores, en un escenario donde las relaciones sociales y la comunicación están 
fuertemente impregnadas por el uso de nuevas tecnologías, constituye una expresión de la vulneración de derechos (Viveros Madariaga, 2001). Es decir, estas personas en tanto sujetos de derecho no acceden per se a procesos educativos que brinden conocimientos para utilizar las nuevas tecnologías, ya que no existen ofertas educativas que los incluyan como destinatarios atendiendo a sus particularidades y necesidades.

La situación descripta podría enmarcarse en lo que Osorio (2006) señala como exclusión social de los ancianos en la compleja sociedad contemporánea, y responde, entre otras causas, al hecho de que ellos se han constituido como "sujetos de beneficio", lo cual los coloca en una posición de receptividad pasiva que nada tiene que ver con su reconocimiento como sujetos de derecho, capaces de asumir un rol activo en el desarrollo y ejercicio de la ciudadanía. Muchos de los estudios consultados demuestran que la realización de este tipo de actividades por parte de personas mayores fomenta el envejecimiento activo y son altamente beneficiosos al mejorar su salud psicosocial, promoviendo la interacción social, la independencia y la autonomía personal que inciden favorablemente en su calidad de vida.

Las tecnologías informáticas actualmente son una herramienta de comunicación, de gestión y de realización de trámites que toma cada vez más preponderancia en la vida de todas las generaciones, frente a las cuales los adultos mayores se encuentran objetivamente en desventaja, al no haber sido socializados en contextos de informatización. Ello exige un trabajo de capacitación que la universidad pública puede ofrecer desde una práctica extensionista que contribuya a producir saberes vinculados a la resolución de situaciones cotidianas. De este modo, las estrategias previstas se focalizan en la cuestión de las nuevas tecnologías y la inclusión digital de las personas mayores.

\section{Programa Abuelos en Red (AER)}

\section{Fundamentación}

En los próximos años, la población de adultos mayores se verá cada vez más limitada para satisfacer sus necesidades de participación en la 
sociedad de la información, si no se instrumentan acciones educativas que los incorporen a procesos de enseñanza y aprendizaje impregnados por tecnología que contribuyan a que la sociedad cambie su mirada sobre este grupo etario.

Se considera que los programas de alfabetización en el uso de tecnologías digitales pueden aportar a mejorar la calidad de vida de los adultos mayores, abriendo canales efectivos de participación social, cultural y de verdadera integración. En este marco se constituye en objetivo central del presente programa desarrollar un proceso de alfabetización en el uso de tecnologías digitales destinado a adultos mayores.

En este sentido, el Banco Supervielle ${ }^{1}$ implementó a partir del año 2007, a través del área de Responsabilidad Social Corporativa, el programa denominado "Abuelos en Red", en él se desarrollan talleres gratuitos de capacitación sobre uso de herramientas informáticas básicas orientadas a la comunicación, destinado para personas mayores de 55 años, que sean jubilados o pensionados. Estos talleres se dictan en distintos espacios, algunas sedes funcionan en Centros de Servicios ${ }^{2}$ del banco y otras en universidades asociadas, los talleres tienen una duración de un mes, el cursado es presencial dos veces por semana de dos horas de duración, con una carga total de 16 horas reloj, luego se realizan acciones de acompañamiento por medio de una página web (www.abuelosenred.org) y en las principales redes sociales.

A medida que se van desarrollado los talleres, los adultos mayores evidencian cambios que se manifiestan notablemente, entre ellos, un mayor interés por la tecnología, exigencia de nuevos talleres, incremento en su participación, progresivo cambio de actitud hacia la adquisición de nuevos conocimientos y la posibilidad de compartir o comunicarse con otros adultos y familiares, inclusive con sus propios nietos.

1 El Banco Supervielle es un entidad financiera con una amplia cobertura territorial y fuerte presencia en la Ciudad Autónoma y la Provincia de Buenos Aires, Córdoba, Mendoza, Santa Fe, Tucumán y San Luis. Posee una red integrada de 168 sucursales y centros de servicios, 272 cajeros automáticos y más de 3.000 empleados, constituyendo el sexto banco privado de capitales nacionales.

2 Los Centros de Servicios son sucursales adaptadas especialmente para la atención de jubilados y pensionados. El Banco Supervielle atiende a más de 1.000 .000 jubilados en el país con su red de Centros de Servicios. 


\section{Objetivos del programa o acción}

Acercar a nuestros adultos mayores al uso de nuevas tecnologías de comunicación e información para promover su rol activo en la sociedad actual.

\section{Descripción del programa o acción}

Abuelos en Red (AER) es el primer programa propio de Responsabilidad Social Corporativa de Banco Supervielle. Lanzado en 2007 en San Luis con la apertura de talleres de informática e internet para adultos mayores, hoy tiene alcance nacional. Creemos en una tercera edad emprendedora y plenamente integrada en su comunidad, que puede encontrar espacios de intercambio generacionales tanto a nivel real como virtual.

El programa se dicta a 35 centros de formación a través de convenios con universidades e institutos de formación públicos y privados, donde los adultos mayores acceden en forma gratuita a ciclos de formación inicial e intermedios en el uso de tecnologías digitales de comunicación e información. Además, cuentan con un sitio exclusivo -www.abuelosenred.org- y una fanspage - Abuelos en Red Supervielle-, en donde pueden participar de cursos a distancia, salas de debate y foros de intercambio.

El programa Abuelos en Red se desarrolla de forma anual de enero a diciembre, desde el año 2007, en las principales universidades del país. Los beneficiarios son adultos mayores de 55 años jubilados o pensionados. Anualmente se llega a más de 15.000 adultos mayores al año con los cursos de formación.

Más de 15.000 abuelos están suscriptos a la página www.abuelosenred.org y 3.000 adultos mayores son seguidores de la fanspage en Facebook Abuelos en Red Supervielle.

El Programa tiene un fuerte impacto territorial, ya que tiene presencia en las siguientes provincias argentinas: Buenos Aires, Córdoba, Tucumán, San Luis, San Juan y Mendoza.

Participan del programa universidades de todo el país, en Buenos Aires: Universidad Nacional de La Plata, la Universidad Tecnológica Nacional Regional Buenos Aires, Universidad Tecnológica Nacional 
Regional General Pacheco, Escuela de Psicología Social de Quilmes, Instituto Superior de Formación y Desarrollo Humano, Universidad de La Matanza, Universidad de Morón, Instituto Padre Mario Pantaleo, Universidad de Flores, Universidad privada de la Fraternidad de Agrupaciones Santo Tomás de Aquino, en la provincia de Santa Fé: Pontificia Universidad Católica Argentina "Santa María de los Buenos Aires”, Facultad de Ciencias Económicas del Rosario, en la provincia de Córdoba: Instituto Cervantes Rio IV, Universidad Tecnológica Nacional Regional Villa Maria, en la provincia de San Luis: Universidad Católica de Cuyo, en la provincia de Mendoza: Pontificia Universidad Católica Argentina "Santa María de los Buenos Aires" Facultad de Ciencias Económicas y Jurídicas, Universidad Champagnat, Universidad Juan Agustín Maza, Universidad del Aconcagua, Instituto Tecnológico Universitario, Universidad de Mendoza, Municipalidad de Lujan de Cuyo, en la provincia de San Juan: Universidad Católica de Cuyo, en la provincia de Tucumán: Universidad del Norte Santo Tomás de Aquino.

Los resultados del programa indican: 35.000 adultos mayores capacitados. 9 años de enseñanza e integración. Más de 1250 talleres de formación.

Más de 20.000 horas capacitación. 24 Universidades Asociadas al programa. 35 Centros de formación. $1^{\circ}$ Premio CCIFA-RSE 2009.

Los resultados en la Facultad de Ciencias Económicas de la UNLP.

El convenio entre el Banco Supervielle y la Facultad de Ciencias Económicas de la UNLP apunta a capacitar a los participantes en informática básica, acceso y utilización de internet (redes sociales, correo electrónico, navegación en internet), así como también en el uso de la página del Banco en la cual pueden consultar actividades y obtener algunos beneficios. Los cursos están destinados a los jubilados y pensionados - mayores de 55 años- que perciban sus haberes previsionales en los Centros de Servicios de Banco Supervielle S.A., ubicados en la ciudad de La Plata.

La Facultad ha ampliado este propósito, dado que en ese marco se formuló el objetivo del programa orientándolo a fortalecer la ampliación de derechos de los adultos mayores mediante prácticas extensionistas que, desde en una estrategia de alfabetización informática, 
contribuyan a producir una mayor inclusión social y a mejorar vínculos entre pares e inter generacionales. No es la primera experiencia de Económicas con adultos mayores. Durante el año 2013, a través de la Federación de Instituciones, la FCE estuvo presente en la Comunidad Rural de Los Hornos, donde además de dictar cursos de iniciación en computación, se inauguró una sala con equipamiento informático.

El proyecto crea las condiciones para permitir que:

- Se produzcan aprendizajes en relación al uso de nuevas tecnologías para posibilitar el acceso de adultos mayores a la construcción de aprendizajes en el uso de las nuevas tecnologías que mejoren la resolución de situaciones cotidianas vinculadas al acceso a bienes y servicios.

- Se fortalezcan las condiciones de inclusión social de esta población mediante la accesibilidad a la alfabetización digital en una sociedad altamente tecnologizada;

- Se amplíe desde la institución universitaria el ejercicio del derecho a la educación mediante esta estrategia extensionista con personas mayores;

- Se genere una apropiación por parte de los integrantes del proyecto, de las herramientas de formación, planificación y gestión de procesos de alfabetización digital con adultos mayores.

La etapa como jubilados inicia con la pérdida de muchos vínculos laborales que se habían sostenido durante gran parte de la vida de la persona. Resulta central entonces construir nuevos vínculos, adaptados a la nueva etapa, que incentiven a seguir relacionándose con otros, asumiendo responsabilidades, creando, comunicándose, conociendo gente nueva de diferentes generaciones y sintiéndose capaces e independientes.

Las tecnologías informáticas actualmente son una herramienta de comunicación que va tomando cada vez más preponderancia en la vida todas las generaciones, pero a la vez resultan muy novedosas para la mayoría de los jubilados. Por eso los cursos para adultos mayores en el uso de tecnologías informáticas pretende formarlos para interactuar de la manera que lo hacen las demás generaciones, mejorar su autoestima 
e integrarlos a nuevos grupos. El curso dura un mes pero una vez terminado siguen abiertos canales de consulta específicos. Más allá de las clases de computación, son muy importantes los almuerzos mensuales que se llevan adelante y las nuevas amistades que surgen espontáneamente de las actividades compartidas. Se promueve un desempeño independiente en el uso de la tecnología informática, para ello cada uno tiene una computadora, se respetan los tiempos de aprendizaje de cada uno, a la vez que se plantean actividades integradoras.

El proceso de trabajo extensionista se estructura a partir de una estrategia metodológica con dos ejes interrelacionados: uno refiere a la formación de formadores, y otro a la producción de condiciones que efectivicen el derecho a la alfabetización digital.

El primer eje, la formación de formadores, supone un proceso de “entreaprendizaje" que procura poner en diálogo y multiplicar los diferentes saberes, aportando a la construcción de reflexiones que fortalezcan el trabajo pedagógico y los aprendizajes en el uso de las Tic. La modalidad de trabajo propuesta es el taller en tanto constituye un espacio de encuentro abocado a la producción colectiva de conocimientos e intercambios. En él, los participantes comparten saberes de manera horizontal, generando momentos de debate que potencian los alcances de la experiencia en lo referente a la producción de herramientas informáticas para ejecutar diversas acciones.

El segundo eje corresponde a la producción de condiciones que efectivicen el derecho a la alfabetización digital mediante acciones educativas dirigidas a favorecer la apropiación de conocimientos y el desarrollo de capacidades y destrezas vinculadas al uso de la tecnología como práctica de inclusión social. Este eje metodológico parte de considerar el lugar de los actores en el proyecto, en tanto sujetos socio-históricos que ponen en juego sus competencias de saber hacer para realizar una práctica de vinculación tecnológica.

La potencialidad de trabajar desde una metodología participativa promueve el compromiso y fortalece la decisión política de los actores que participan del proceso, en pos de generar condiciones de mayor inclusión social para los adultos mayores. El producir condiciones de realización de una práctica como el uso cotidiano de las nuevas tecnologías informáticas, constituye una clara estrategia de ampliación y 
reconocimiento de derechos, y nos ubica en un plano de mayor proximidad para generar acuerdos sobre los contenidos y formatos de capacitación, sobre los intereses y necesidades de los destinatarios, así como respecto de los espacios de circulación y del sentido de transformación que plantea la estrategia extensionista. Los adultos mayores participan de reuniones de gestión y evaluación en proceso del proyecto.

\section{Conclusiones}

Las redes interorganizacionales presuponen un campo de acción constituido por organizaciones independientes que se han distribuido las tareas y se articulan de una manera tal que obtienen los objetivos compartidos de una política. Esta interacción lleva a encontrar oportunidades que generan valor público de una manera poco costosa. Este trabajo es una evidencia de lo que se denomina Responsabilidad Social Interorganizacional (RSI), una red vincular entre organizaciones públicas, privadas y organizaciones de la sociedad civil, construida para potenciar las capacidades de los participantes en pos de lograr objetivos compartidos de una política de responsabilidad social.

Las alianzas entre organizaciones públicas, privadas y organizaciones de la sociedad civil, en su caso, se constituyen en dinamizadores de la responsabilidad social organizacional, y se convierten en oportunidades de satisfacer y anticipar las necesidades de los distintos grupos de interés, permeando las fronteras del ámbito de una organización en particular. Los vínculos generados entre el Banco y la Universidad nucleados en sus Políticas de Responsabilidad Social potencian los impactos de un programa iniciado por una institución privada, orientado a la alfabetización informática de los adultos mayores que encuentra en la universidad su socio estratégico.

A su vez, esto permite desde la institución universitaria el ejercicio del derecho a la educación mediante una estrategia extensionista y que se genere una apropiación por parte de los integrantes del proyecto, de las herramientas de formación, planificación y gestión de procesos de alfabetización digital con adultos mayores. Si esta tarea se lleva cabo en un marco de análisis y discusión amplia y participativa, con 
Responsabilidad Social de las Organizaciones (RSO)

intervención de los múltiples actores involucrados, con espíritu tendiente a la búsqueda de consensos, quizás interpretar más cabalmente el presente y diseñar los escenarios del futuro sea más factible y se logren acuerdos de mayor significación. 


\section{Referencias}

Cammarota, E. y Barenblum, D. (2009). Responsabilidad Social Empresarial y Gerencia Social: Partícipes necesarios para el Desarrollo Humano. En D. Stolar y E. Stolar (Comps.), Responsabilidad Social Empresaria. Buenos Aires: Valletta Ediciones SRL.

Cunill Grau, N. (1999). Repensando lo público a través de la sociedad. Nuevas formas de gestión pública y representación social. Caracas, Venezuela: Ed. Nueva Sociedad.

Dominguez Granda, J.B. (2015). Fuentes de la Responsabilidad Social, Perú. III Sirso. "Responsabilidad Social de las Organizaciones, avances y propuestas en América Latina”, Ed. Udalech.

Jaramillo, M. (2006). Redes sociales interorganizacionales. Documento técnico para "La Iniciativa de Formación en Gerencia Social para la Protección Social”. Zona Sur-Occidente Fonade. Universidad Icesi. Redes Sociales e Interorganizacionales.

Kliksberg, B. (2003). Hacia una nueva visión de la política social en América Latina. Desmontando mitos. Revista Venezolana de Gerencia, Año 8, N. ${ }^{\circ} 21$, pp. 9-37.

Martínez, N., Acuna, R. y Tuzzi, M.J. (2014). Adultos mayores, inclusión social y autonomía. III Jornada de Extensión del Mercosur, Unicen, Argentina.

Mayntz, R. (2001). El Estado y la sociedad civil en la gobernanza moderna. Revista del CLAD, Reforma y Democracia, N. ${ }^{\circ} 21$. Caracas.

Messner, D. (1995). La sociedad de redes. Alemania: Colonia.

OEA-BID (2007). ¿Cómo enseñar ética, capital social y desarrollo en la universidad? Estrategias de RSU. Washington: OEA-BID.

Osorio, P. (2006). Exclusión generacional: la tercera edad. Revista Mad, 14, 7-12. Departamento de Antropología, Universidad de Chile.

Vallaeys, F. (2008). Breve marco teórico de la responsabilidad social universitaria. Lima, BID-Universidad Católica del Perú.

Vallaeys, F. (2009). Responsabilidad Social Universitaria. Manual de primeros pasos. McGraw Hill.

Viveros Madariaga, A. (2001). Cepal. Serie Población y Desarrollo N. 22. Santiago de Chile.

Volpentesta, R. (2009). Gestión de la Responsabilidad Social Empresarial. Argentina: Editorial. Osmar D.. 
